04, 10,09

\title{
Рамановское рассеяние кристаллами гексаборидов редких земель с различными изотопами бора
}

\author{
(C) Ю.Ф. Марков ${ }^{1}$, В.Н. Гурин ${ }^{1}$, К.В. Понкратов ${ }^{2}$ \\ ${ }^{1}$ Физико-технический институт им А.Ф. Иофрее РАН, \\ Санкт-Петербург, Россия \\ ${ }^{2} \mathrm{OOO}$ „Ренишоу“, \\ Москва, Россия \\ E-mail: Yu.marhov@mail.ioffe.ru
}

(Поступила в Редакцию 10 октября 2017 г.)

Раствор-расплавным методом получены монокристаллы гексаборида лантана $\mathrm{LaB}_{6}$, содержащие как природный бор, так и его изотопы ${ }^{10} \mathrm{~B}$ и ${ }^{11} \mathrm{~B}$. Впервые выращены полиэлементные гексабориды редких земель и синтезированы соответствующие керамики. Все эти кристаллы изучены при помощи различных методик, главным образом, при помощи рамановского рассеяния. Получены и интерпретированы рамановские спектры с приписанием различных линий спектра соответствующим неприводимым представлениям. Получены частоты и полуширины линий спектра, обнаружено индуцированное дефектами, главным образом, присутствием различных изотопов бора, снятие вырождения и проявление соответствующих расщеплений вырожденных колебаний. Определено влияние дефектов на рамановские спектры.

DOI: $10.21883 /$ FTT.2018.04.45681.282

\section{1. Введение}

Из-за необычных физических и химических свойств гексабориды $M \mathrm{~B}_{6}$ имеют важные практические приложения. Они обладают высокой твердостью, химически инертны и являются огнеупорными материалами. Гексабориды, содержащие трехвалентные элементы $(M=\mathrm{La}$, $\mathrm{Nd}, \mathrm{Gd}, \mathrm{Tb}, \mathrm{Dy})$, обычно являются металлическими проводниками, а содержащие двухвалентные элементы $(M=\mathrm{Ca}, \mathrm{Sr}, \mathrm{Eu}, \mathrm{Sm}, \mathrm{Yb})$ - полупроводниками $[1,2]$. Соединения лантана $\mathrm{LaB}_{n}(n=2,4,6 \ldots)$ являются сверхпроводниками при низких температурах [3] и используются в технике в качестве термоэлектронных эмиттеров (при $n=6$ ).

Гексабориды кристаллизуются в простой кубической структуре типа $\mathrm{CsCl}$ (пространственная группа $\left.P m \overline{3} m=O_{h}^{1}[4]\right)$. Катион занимает центр куба, а октаэдры $\mathrm{B}_{6}$ находятся в углах куба (рис. 1).

Изучению колебательных спектров гексаборидов ранее уделялось относительно мало внимания, прежде всего, из-за проблем, связанных с подготовкой образцов. Спектры инфракрасного излучения трудно изучать, поскольку образцы для измерений не могут быть легко приготовлены как в виде мелкодисперсных порошков, так и в виде подходящих и очень тонких ориентированных монокристаллических пластин. Монокристаллы гексаборидов имеют обычно темный цвет, от фиолетового до черного, что делает их плохими и трудоемкими объектами для изучения рамановских спектров. Тем не менее как инфракрасные, так и рамановские спектры этих соединений были успешно получены с помощью современных Фурье- и многоканальных спектрометров $[5,6]$.

В настоящей работе были впервые выращены монокристаллы гексаборидов лантана $\mathrm{LaB}_{6}$, синтезированные как на природном боре $\left({ }^{11} \mathrm{~B} 80.2 \%,{ }^{10} \mathrm{~B} 19.8 \%\right)$, так и на его изотопах ${ }^{10} \mathrm{~B}$ и ${ }^{11} \mathrm{~B}$, а также полиэлементные кристаллы гексаборидов $\mathrm{La}_{0.5} \mathrm{Ce}_{0.1} \mathrm{Pr}_{0.1} \mathrm{Nd}_{0.1} \mathrm{Sm}_{0.1} \mathrm{Eu}_{0.1} \mathrm{~B}_{6}$, содержащих одновременно 6 редкоземельных элементов, и соответствующие керамики этих полиэлементных гексаборидов. Последние соединения были впервые получены с целью изучения и расширения практического использования гексаборидов редких земель.

Полиэлементная керамика (те же 6 элементов, что и в монокристаллах) была синтезирована в Технологическом институте-университете (СПбГТУ) для напыления
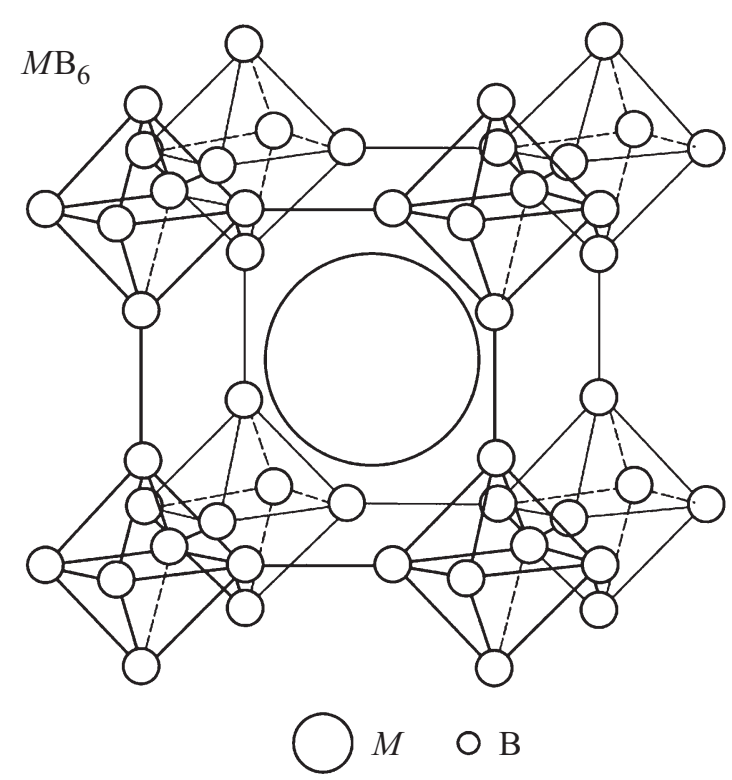

Рис. 1. Кубическая элементарная ячейка гексаборидов $M \mathrm{~B}_{6}$ (M- редкие земли). 
Частоты полуширины $\left(\mathrm{cm}^{-1}\right)$ и интерпретация основных линий рамановских спектров кристаллов гексаборидов лантана

\begin{tabular}{|c|c|c|c|}
\hline \multirow[b]{2}{*}{ Симметрия } & \multicolumn{3}{|c|}{ Гексабориды } \\
\hline & $\begin{array}{c}\mathrm{La}^{10.81} \mathrm{~B}_{6} \text { (естественное } \\
\text { содержание изотопов бора) }\end{array}$ & $\mathrm{La}^{10} \mathrm{~B}_{6}$ & $\mathrm{La}^{11} \mathrm{~B}_{6}$ \\
\hline $\begin{array}{l}\text { Рамановские спектры } \\
\text { второго порядка }\end{array}$ & $207(18)$ & $207(20)$ & $\begin{array}{l}207(22) \\
588\end{array}$ \\
\hline$T_{2 g}$ & $\begin{array}{l}674 \text { (20). На самом деле, } \\
\text { это дублет: } 672(13), 683 \text { (29) }\end{array}$ & $\begin{array}{l}695 \text { (25). На самомо деле, } \\
\text { это дублет: } 691(18), 699(27)\end{array}$ & $\begin{array}{l}675(45)-\text { идеальная } \\
\text { симметрия (Гаусс) } \\
986\end{array}$ \\
\hline$E_{g}$ & 1116, 1155 - дублет & 1157, 1197 - дублет & 1095,1153 - дублет \\
\hline$A_{1 g}$ & $\begin{array}{l}1244 \text { (22) линия с максимальной } \\
\text { интенсивностью }\end{array}$ & $1280(19)$ & $1230(32)$ \\
\hline $\begin{array}{l}\text { Рамановские спектры } \\
\text { второго порядка }\end{array}$ & $1383(150)$ & $1430(85)$ & $1376(120)$ \\
\hline
\end{tabular}

Примечан ие. В скобках приводятся полуширины линий рамановских спектров.

пленок с целью выбора материала для термоэлектрического тестера рентгеновского и ультрафиолетового излучения в однофотонном приближении. Керамика и монокристаллы имеют одинаковый период решетки, в этом составе она получена впервые.

Существовала необходимость сопровождения наших технологических экспериментов обнаружением и изучением изотопов ${ }^{10} \mathrm{~B}$ и ${ }^{11} \mathrm{~B}$. В этом случае весьма подходящей методикой служит рамановская спектроскопия, позволяющая фиксировать весьма тонкие изменения состава и структуры объектов исследования, а также получать информацию о качестве и степени дефектности синтезированных соединений.

В настоящей работе были получены, изучены и интерпретированы рамановские спектры этих гексаборидов, в том числе и полиэлементных, с целью диагностики и получения информации о динамике кристаллических решеток, симметрии и микроструктуре синтезированных образцов.

\section{2. Методика эксперимента}

Монокристаллы вышеуказанных соединений получали из раствора-расплава алюминия по методике $[7,8]$ : навески редкоземельного металла и бора помещали в расплав алюминия, нагревали $2-3 \mathrm{~h}$ до $1400^{\circ} \mathrm{C}$, выдерживали $4 \mathrm{~h}$ и охлаждали с ,ускорением“ до $600^{\circ} \mathrm{C} \quad 6-12 \mathrm{~h}$. Полученные слитки растворяли в разбавленной соляной кислоте $(1: 2-1: 4)$. Из образовавшегося осадка отбирали кристаллы и подвергали их рентгеноструктурной диагностике, а затем прецизионным спектроскопическим исследованиям (рамановское рассеяние). Способ получения монокристаллов с различными изотопами бора ничем не отличался от такового с природным бором.

Спектры рамановского рассеяния в области $100-1500 \mathrm{~cm}^{-1}$ регистрировались на спектрометре Renishaw, оборудованном охлаждаемой жидким азотом 2DCCD-камерой. B качестве источника возбуждения использовали вторую гармонику непрерывного $\mathrm{Nd}$ :YAG-лазера с длиной волны излучения $532 \mathrm{~nm}$. Для фокусировки и сбора рассеянного излучения применяли микроскоп с объективом $50 \times$, размер пятна на образце не превышал 1-2 mm. Время интегрирования сигнала $\sim 0.3-10 \mathrm{~s}$ в зависимости от размеров и качества образцов и интенсивности рамановских спектров.

В случае кристаллов гексаборидов металлов, в том числе и редких земель, теоретико-групповой анализ $[5,6]$ дает следующий набор оптических колебаний

$$
\Gamma_{\mathrm{opt}}=A_{1 g}+E_{g}+T_{1 g}+T_{2 g}+2 T_{1 u}+T_{2 u} .
$$

В рамановских спектрах должны быть активны четные колебания симметрии $A_{1 g}, E_{g}$ и $T_{2 g}$, а в инфракрасных (в отражении или в поглощении) - нечетные колебания симметрии $T_{1 u}$; колебания $T_{1 g}$ и $T_{2 u}$ запрещены в обоих спектрах. В рассматриваемых центросимметричных кристаллах должно иметь место правило альтернативного запрета - четные фононы наблюдаются только в рамановских спектрах и не проявляются в ИК спектрах, и наоборот, нечетные - только в ИК спектрах и запрещены в рамановских спектрах. В принципе, эти правила могут быть частично нарушены различными дефектами, локально понижающими симметрию кристаллов и индуцирующими проявление в спектрах запрещенных по симметрии малоинтенсивных фононов.

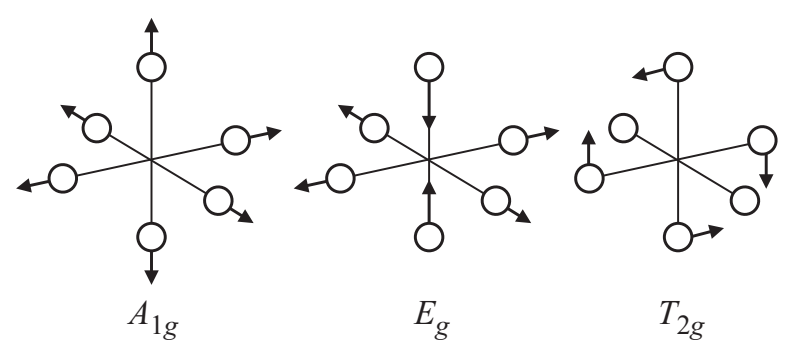

Рис. 2. Собственные вектора (нормальные колебания), соответствующие раман-активным фононам в кристаллах $\mathrm{LaB}_{6}$. 


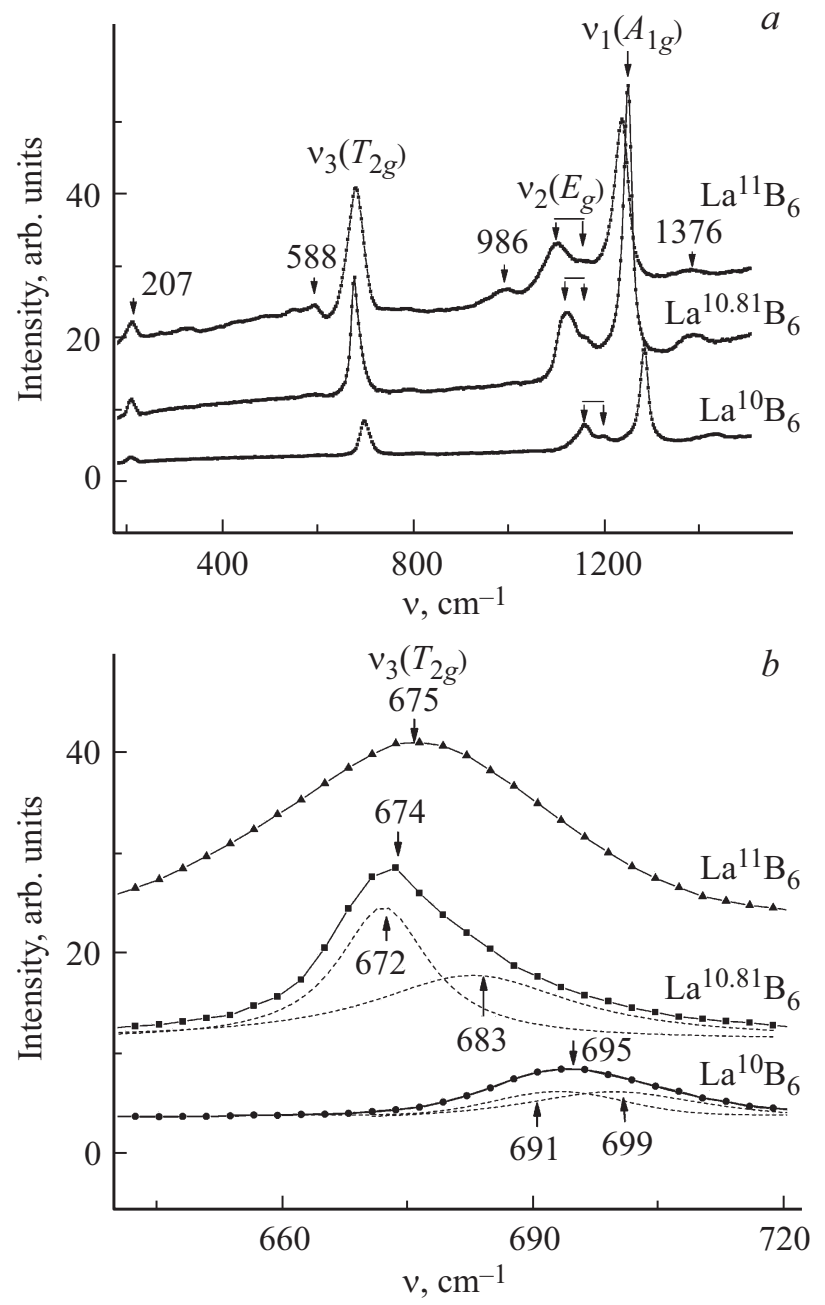

Рис. 3. Рамановские спектры кристаллов гексаборидов лантана, содержащие различные изотопы бора $(a)$ и кристаллов $\mathrm{LaB}_{6}$ в области трижды вырожденных колебаний $T_{2 g}$ с разложением на исходные лоренцианы (штриховые линии) $(b)$.

Собственные вектора (нормальные колебания), соответствующие раман-активным фононам, приведены на рис. 2. Можно видеть, что все смещения атомов бора симметричны относительно центра инверсии кристалла, что и должно иметь место.

На рис. 3, $a$ приведены рамановские спектры кристаллов чистых гексаборидов $\mathrm{LaB}_{6}$, содержащих различные изотопы бора, в том числе естественный природный бор, являющийся смесью ${ }^{11} \mathrm{~B}$ и ${ }^{10} \mathrm{~B}$ с атомным весом 10.81 , а также спектры гексаборидов, содержащих только ${ }^{10} \mathrm{~B}$ или ${ }^{11} \mathrm{~B}$. Основные характеристики (параметры) этих спектров (частоты фононов, полуширины линий и интерпретация) приведены в таблице.

В этих спектрах присутствуют несколько интенсивных линий, соответствующих полносимметричным колебаниям (фононам) $A_{1 g}$, дважды вырожденным колебаниям симметрии $E_{g}$ и трижды вырожденным колебаниям симметрии $T_{2 g}$. Интерпретация наиболее интенсивных линий спектра вполне очевидна и основана на совпадении численных расчетов и экспериментальных спектров $[9,10]$. Полуширины этих линий достаточно большие, так как, например, в случае вырожденных колебаний в естественных гексаборидах $\mathrm{La}^{10.81} \mathrm{~B}_{6}$ они обременены также частичным снятием вырождения в спектрах из-за наличия в каждом „боровском“ октаэдре по крайней мере одного ${ }^{10} \mathrm{~B}$ из шести. В этом случае автоматически в октаэдрах реализуется понижение симметрии от кубической до тетрагональной, ромбической или до более низкой - моноклинной.

Из рис. 3, $a$ можно видеть эти понижения симметрии, когда каждое дважды вырожденное колебание (симметрии $E_{g}$ ) расщепляется на дублет (обозначен квадратной скобкой). Трижды вырожденное колебание $T_{2 g}$ также, по крайней мере, состоит из двух компонент (см. разложение асимметричного контура на два Лоренциана штриховые линии на рис. $3, b$ ). В спектре гексаборида $\mathrm{La}^{10} \mathrm{~B}_{6}$ также надежно наблюдаются расщепления вырожденных колебаний, но индуцированные как присутствием небольшого количества других изотопов бора, так и достаточно большим количеством неконтролируемых дефектов.

В случае гексаборида $\mathrm{La}^{11} \mathrm{~B}_{6}$ в явном виде аналогичные расщепления трижды вырожденного колебания $T_{2 g}$ надежно наблюдать не удается (рис. $3, b$ ), что, возможно, скрыто в бо́льшей полуширине линий спектра по сравнению с другими гексаборидами (рис. $3, a, b$ ).

Остальные менее интенсивные линии спектра можно приписать, но достаточно неоднозначно, спектрам второго порядка, т.е. комбинациям четных $(g x g=g)$ или нечетных $(u x u=g)$ фононов, что дает четный фонон, активный в рамановских спектрах. Достаточно узкая и малоинтенсивная линия с частотой $207 \mathrm{~cm}^{-1}$ может быть приписана проявлению разностных фононов [9], хотя этот наблюдаемый и значительно более узкий максимум напоминает проявление какого-то локального (квазилокального) колебания, возможно, связанного с образованием каких-либо кластеров. Из-за понижения локальной симметрии (в том числе из-за потери центра инверсии) мог бы проявиться и ИК активный фонон в области $1100 \mathrm{~cm}^{-1}[5]$, который, возможно, и присутствует в этой области частот рамановского спектра. Линия $1385 \mathrm{~cm}^{-1}$ также в ряде работ интерпретирована (спектры II порядка), что, по-видимому, соответствует действительности [9].

Все приведенные на рис. $3, a, b$ рамановские спектры гексаборидов лантана, содержащих различные изотопы бора ${ }^{10.81} \mathrm{~B},{ }^{10} \mathrm{~B}$ и ${ }^{11} \mathrm{~B}$, в основных чертах похожи, но есть и принципиальные отличия: в последовательности $\mathrm{La}^{11} \mathrm{~B}_{6}, \quad \mathrm{La}^{10.81} \mathrm{~B}_{6}, \quad \mathrm{La}^{10} \mathrm{~B}_{6}$ имеет место убедительный сдвиг частот фундаментальных колебаний $A_{1 g}, E_{g}$ и $T_{2 g}$ в сторону более высоких значений, на $30-10 \mathrm{~cm}^{-1}$ для колебаний различных симметрий, чего, в принципе, и следовало ожидать, так как изотоп бора с массой 11 замещается на более легкий изотоп с массой 10 . При учете только этого дефекта масс в случае чисто валентных колебаний атомов бора эти смещения могли бы 


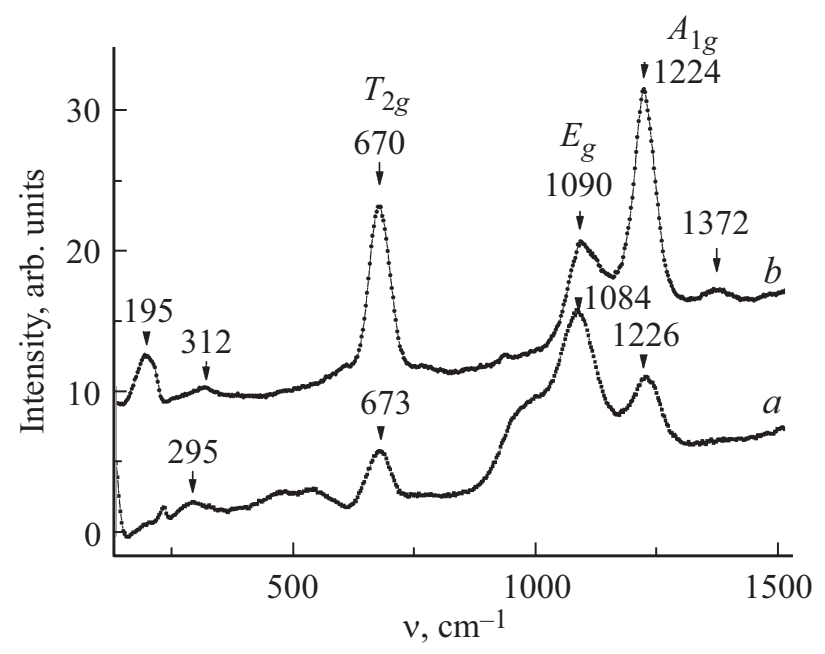

Рис. 4. Рамановские спектры кристаллов полиэлементных гексаборидов редких земель $\mathrm{La}_{0.5} \mathrm{Ce}_{0.1} \mathrm{Pr}_{0.1} \mathrm{Nd}_{0.1} \mathrm{Sm}_{0.1} \mathrm{Eu}_{0.1} \mathrm{~B}_{6} \quad(a)$ соответствующих керамик $(b)$.

достигать $\sim 50 \mathrm{~cm}^{-1}$, исходя лишь из простой оценки сдвига частот по формуле $v \sim \sqrt{k / \mu}$, где $k-$ силовая константа, $\mu$ - приведенная масса колеблющихся атомов. Как видно, эксперимент и теоретические оценки не противоречат друг другу.

Обсуждая полуширины основных линий спектра, можно отметить, что минимальные полуширины линий проявляются в случае кристаллов с природным бором и изотопом бора ${ }^{10} \mathrm{~B}$, что может указывать на достаточно высокое качество этих гексаборидов. Образцы $\mathrm{La}^{11} \mathrm{~B}_{6}$ демонстрируют в спектрах несколько размытую картину с проявлением бо́льшего количества малоинтенсивных линий. Возникает предположение, что последние кристаллы более дефектны, однако снятие вырождения для колебаний $T_{2 g}$ явно в них не наблюдается, хотя оно, по-видимому, „спрятано“ в большой полуширине линии этого колебания. Этот вывод достаточно аргументирован, так как в этих кристаллах $\mathrm{La}^{11} \mathrm{~B}_{6}$ убедительно проявляется расщепление дважды вырожденных колебаний симметрии $E_{g}$.

На рис. 4, $a, b$ приведены типичные (характерные) рамановские спектры смешанных кристаллов полиэлементных гексаборидов редких земель $\mathrm{La}_{0.5} \mathrm{Ce}_{0.1} \mathrm{Pr}_{0.1} \mathrm{Nd}_{0.1} \mathrm{Sm}_{0.1} \mathrm{Eu}_{0.1} \mathrm{~B}_{6}$, а также спектры соответствующих керамик. В первом случае мы имеем дело с редкоземельными твердыми растворами гексаборидов, а во втором - с керамиками этих соединений, приготовленными по специальной технологии и имеющими тот же самый химический состав и тот же период кристаллической решетки. Видно, что имеется удовлетворительное соответствие спектров твердых растворов и спектров монокристаллов чистого $\mathrm{LaB}_{6}$. Отметим также бо́льшую полуширину линий фононов симметрии $A_{1 g}, E_{g}$ и $T_{2 g}$ относительно этих линий в спектре соответствующих керамик. Значительно меньшую полуширину линий в последнем случае можно связать лишь с достаточно длительной термообработкой, главным образом, с высокотемпературным отжигом керамических образцов.

Все изученные гексабориды несколько неоднородны по структуре, составу и локальной симметрии. Каждый образец (монокристалл) гексаборидов, использованный в настоящей работе, изучали в нескольких различных точках, когда имела место удовлетворительная воспроизводимость спектров, однако наблюдались и некоторые различия в рамановских спектрах как по интенсивности, так и по частоте и полуширине линий фононов. Разброс значений частот в соседних точках одного и того же образца достигал $2-3 \mathrm{~cm}^{-1}$. Как указывалось в работе [10], гексабориды содержат большое количество дефектов, в том числе значительное количество катионных (анионных) вакансий (до 2-3\%), наличие которых понижает локальную симметрию кристаллов от кубической до тетрагональной, ромбической и т.д., а также может приводить к нарушению центросимметричности этих кристаллов и соответственно к нарушению альтернативных правил отбора, когда в рамановских спектрах могут проявляться и нечетные ИК активные фононы (в частности, в области $1100 \mathrm{~cm}^{-1}$ [5]), но этот эффект однозначно в наших экспериментах наблюдать не удалось.

Авторы выражают признательность участникам технологических экспериментов по получению монокристаллов гексаборидов редкоземельных металлов (М.М. Корсукова) и полиэлементной керамики (С.С. Орданьян).

\section{Список литературы}

[1] Редкоземельные металлы и их соединения. Наук. думка, Киев (1970).

[2] Редкоземельные полупроводники. Наука, Л. (1977).

[3] B.T. Matthias, T.H. Geballe, K. Andress, E. Gorenzwit, G.W. Hull, J.P. Maita. Science 159, 530 (1968).

[4] I. Morke, V. Dvorak, P. Wachter. Solid State Commun. 40, 331 (1981).

[5] Z. Yahia, S. Turrell, G. Turrell, J.P. Mercurio. J. Mol. Struct. 224, 303 (1990).

[6] T.M. Mattox, S. Chockkalingam, I. Roh, J.J. Urban. J. Phys. Chem. C 20, 9, 5188 (2016).

[7] V.N. Gurin, M.M. Korsukova. Prog. Cryst. Growth Charact. 6, 59 (1983).

[8] В.Н. Гурин, М.М. Корсукова, ЖВХО 26, 6, 79 (1981).

[9] N. Ogito, S. Nagai, N. Okamoto, M. Odagava, F. Iga, M. Sera, J. Akimitsu, S. Kunii. Phys. Rev. B 68, 224305 (2003).

[10] Z. Yahia, S. Turrell, J.P. Mercurio, G. Turrell. J. Raman Spectroscopy 24, 207 (1993). 